\title{
Increasing the Efficiency of Rake Receivers for Ultra-Wideband Applications
}

\author{
Aimilia P. Doukeli, Athanasios S. Lioumpas, Student Member, IEEE, \\ George K. Karagiannidis, Senior Member, IEEE, Panayiotis V. Frangos, Senior Member, IEEE \\ and P. Takis Mathiopoulos, Senior Member, IEEE
}

\begin{abstract}
In diversity rich environments, such as in UltraWideband (UWB) applications, the a priori determination of the number of strong diversity branches is difficult, because of the considerably large number of diversity paths, which are characterized by a variety of power delay profiles (PDPs). Several Rake implementations have been proposed in the past, in order to reduce the number of the estimated and combined paths. To this aim, we introduce two adaptive Rake receivers, which combine a subset of the resolvable paths considering simultaneously the quality of both the total combining output signal-to-noise ratio (SNR) and the individual SNR of each path. These schemes achieve better adaptation to channel conditions compared to other known receivers, without further increasing the complexity. Their performance is evaluated in different practical UWB channels, whose models are based on extensive propagation measurements. The proposed receivers compromise between the power consumption, complexity and performance gain for the additional paths, resulting in important savings in power and computational resources.
\end{abstract}

Keywords-Adaptive Rake receivers, diversity techniques, fading channels, UWB channel.

\section{INTRODUCTION}

Ultra-Wideband (UWB) systems have attracted great research interest since early 90's. One of the key advantages of UWB signals is the immunity to fading, since the bandwidth of several gigahertz improves the capability of resolving multipath components (MPCs). By incorporating Rake receivers, which have the ability to extract and individually process several signal multipath components, the performance and reliability of wireless communication systems can be significantly improved.

The optimal diversity combining scheme, in terms of performance, is the all-Rake (ARake) receiver which combines all the resolvable paths [1]-[4]. However the performance gain comes at the cost of power consumption and increased complexity of the utilized hardware, which are significant factors in environments with more than $100 \mathrm{MPCs}$, such in UWB applications [5]. A Rake receiver that overcomes these obstacles at the cost of performance is the selective Rake (SRake) receiver [1]-[4]. SRake combines the $L_{b}$ strongest resolvable paths, but still requires full estimation of the

A. P. Doukeli and P. V. Frangos are with the School of Electrical and Computer Engineering, National Technical University of Athens, Greece (e-mail: doukeli@mail.ntua.gr,pfrangos@central.ntua.gr.)

A. S. Lioumpas and G. K. Karagiannidis are with the Department of Electrical and Computer Engineering, Aristotle University of Thessaloniki, 54124 Thessaloniki, Greece (e-mail: \{alioumpa;geokarag\}@auth.gr).

P. T. Mathiopoulos is with the Institute for Space Applications and Remote. Sensing, National Observatory of Athens, 15236 Athens, Greece (e-mail: mathio@space.noa.gr). channel coefficients which may not always be available. Recently, partial Rake (PRake) receiver was proposed in [6]. PRake combines only the first arriving $L_{p}$ paths out of the available resolvable MPCs, and therefore requires only synchronization, but not full channel estimation.

Both SRake and PRake receivers have a fixed processing complexity, since the number of the combined paths is predetermined (i.e., $L_{p}$ paths are combined). This fact raises some disadvantages regarding the compromise between complexity and performance. More specifically, SRake may combine unnecessarily too many paths (e.g. in channels with strong multipaths), or weak paths that do not contribute to increasing the signal's quality. Similarly, PRake may also combine unnecessarily too many paths, but it could also combine insufficient number of paths, since it resolves only the first arriving $L_{p}$ paths, without guaranteeing the desired quality. These issues are important for communication systems operating in wireless channels with intense power delay profiles (PDPs), such as the UWB channel, where the number of resolvable paths is extremely large.

Alternative Rake implementations that aim to reduce the number of the estimated and combined paths, include the generalized selection combining (GSC) receivers that are divided into two categories: in the first one the combined paths are determined by the signal-to-noise ratio (SNR) of each individual path (absolute threshold GSC (AT-GSC), normalized threshold GSC (NT-GSC) [7]), while in the latter one the same decision is based on the combiner's output SNR (minimum selection GSC (MS-GSC), output threshold GSC (OT-GSC), minimum estimation and combining GSC (MEC-GSC), [8]- [12]). However, in Rake receivers of the former category the selected branches may be unnecessarily too many, while a sufficient quality of communication could be possibly attained with less, especially in environments with strong multipaths. Similarly, the Rake receivers of the latter category could keep adding weak paths (e.g. in diversity rich environments with strong PDPs) in an attempt to reach the predetermined threshold, while a slightly worse or the same performance could be achieved by combining only the strong branches.

Motivated by the advantages that each of the above mentioned receivers offer, we propose an Adaptive Selective Rake (ASRake), combining the paths that satisfy simultaneously two predetermined criteria: the quality of each individual path and the quality of the expected combined output signal. More specifically, ASRake keeps adding the strongest paths in order to reach the predetermined quality 
of service (QoS), unless it estimates that the addition of another path will not compensate the expected performance improvement. In this way, the receiver compromises between complexity and performance, since it achieves the best possible performance with the least necessary combined paths.

Furthermore, we propose an Adaptive Partial Rake (APRake) receiver with similar operation as that of ASRake, but now the resolvable paths are not ranked with respect to their signal powers. By evaluating the error performance of the ASRake and APRake receivers in realistic UWB channels [6], it is shown that the improved adaptation leads to important savings in power and computational resources. Thus, the contribution of this work is twofold. First, we propose two novel adaptive Rake receivers, which offer important savings in power and computational resources when operating in realistic UWB channels, compared to previously proposed receivers. Second, we evaluate the performance of several Rake receivers in practical UWB channels, which have been proved to have considerable differences from the narrowband wireless channels [13].

The remainder of the paper is organized as follows. The channels employed in the analysis are briefly described in Section II. In Section III, we present the mode of operation of the proposed receiver, while its performance is evaluated in Section IV. Finally, some concluding remarks are presented in Section V.

\section{Channel Models}

The differences between the UWB and the narrowband wireless channel is are important, especially when the fading statistics and the time of arrival of the MPCs are considered. These differences raise from the fact that the UWB systems cover a bandwidth of almost $10 \mathrm{GHz}$, generating new effects, which make the central limit theorem not applicable and therefore the amplitude of fading statistics are not Rayleigh. [13]. However, previous performance analyses of UWB systems assumed Rayleigh fading, owing to the lack of a suitable channel model.

The most suitable channel models for practical UWB applications are the low-frequency (LF) and the high-frequency (HF) models [6]. The former was accepted by the IEEE 802.15.4a standardization group and is appropriate for applications below $1 \mathrm{GHz}$. The latter was accepted by the IEEE 802.15.3a standardization group and is used in high-datarate UWB communications systems. Next, we evaluate the performance of the proposed receivers over the LF, HF and Rayleigh channels. Details concerning these UWB channel models can be found in [6]. In the following, the main characteristics of these models are given for the reader's convenience.

\section{A. LF Channel Model}

The LF UWB channel model is based on experimental data collected in a typical office building using baseband pulses with duration $1 \mathrm{nsec}$ with a resulting bandwidth of $500 \mathrm{MHz}$ [14]- [17]. The channel's PDP is characterized as a stochastic tapped-delay line model, where the $k$ th tap is determined by the time delay, $\tau_{k}=2(k-1)$, and the path gain $G_{k}$, which is the superposition of large and small scale fading. In the small-scale region, the $G_{k}$ are random variables, with a probability density function (pdf) that can be approximated by a Gamma distribution, with mean $\bar{G}_{k}$ and shape parameter $m_{k}$. The values of $\bar{G}_{k}$ are specified in $\left[6,\left(\right.\right.$ Eq. 1)], while $m_{k}$ are Gaussian-distributed random variables.

\section{B. HF Channel Model}

The HF UWB channel model is based on the SalehValenzuela channel model [18] and is intended to represent the channel characteristics in the frequency range from 3.1 to $10.6 \mathrm{GHz}$ [19]. According to this model, the received signal rays arrive in $L$ clusters each containing $K$ rays. The channel impulse response of the $i$-th realization is defined as

$$
h_{i}(t)=X_{i} \sum_{l=0}^{L} \sum_{k=0}^{K} a_{k, l}^{i} \delta\left(t-T_{l}^{i}-\tau_{k, l}^{i}\right)
$$

where $a_{k, l}^{i}$ is the tap weight associated with the $k$-th ray of the $l$-th cluster, $X_{i}$ is the log-normal shadowing and $T_{l}^{i}, \tau_{k, l}^{i}$ are the cluster and ray arrival times, respectively. Compared to the LF channel mode, the HF model has two important differences; the arrival statistics of the MPCs and the distribution of their amplitudes. More specifically, the LF channel is more dense, i.e. the rays are almost continuous with an exponential decay, while in the HF channel the PDP is more sparse, in the sense that many paths may not carry any energy, which means that the first arriving path is not necessarily the strongest one. Regarding the distribution of the MPCs' amplitude, in the HF model MPCs follow a lognormal distribution with variance that is independent of the path delays. On the other hand, in the LF model, the MPCs distribution is the Nakagami whereas the $m$ parameters decrease with delay.

\section{System Model ANd Mode OF OPERATION}

Regardless of the statistical model assumed, the general form of the UWB channel response has the following mathematical form

$$
h(t)=\sum_{m=0}^{M-1} h_{m} \delta\left(t-\tau_{m}\right)
$$

where $h_{m}$ denotes the channel gain of the $m$-th resolvable path out of $M$ available ones and $\tau_{m}$ is the arrival delay with respect to the first arriving path. The statistics of both $h_{m}$ and $\tau_{m}$ are determined by the channel model that is utilized (e.g. the HF channel model). We consider a Rake receiver, which is assumed to be capable of ideally (i.e. no channel or delay estimation errors occur at the receiver) capturing the energy of the $M$ paths, and combines them using maximalratio combining (MRC). We note, that these assumptions have been followed in the majority of previously published works and leads to the study of the optimum lower bound of the error rate performance. The receiver actually sums 


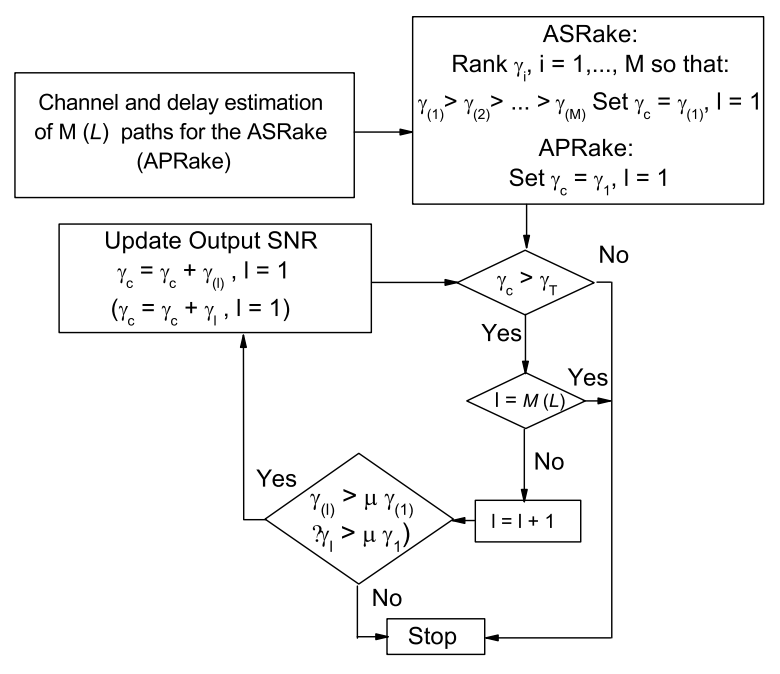

Fig. 1. ASRake and APRake: mode of operation.

the SNR of the resolvable paths, so that the total combined signal has a SNR

$$
\gamma_{\text {total }}=\sum_{p \in R_{M}} \gamma_{p}=\sum_{p \in R_{M}} E_{s} \frac{h_{p}}{N_{0}}
$$

where $R_{M}$ is the subset of the paths that are combined and depends on the Rake receiver that is applied (e.g. for ARake $R_{M}$ involves all the $M$ paths), $\gamma_{p}$ is the SNR of the $p$-th path of subset $R_{M}, E_{s}$ denotes the transmitted symbol's energy, $h_{p}$ stands for the gain of the $p$-th path and $N_{0}$ is the power spectral density of the additive white Gaussian noise (AWGN).

The characteristic that diversifies the Rake receivers is the number of the estimated and combined branches. For example, ARak estimates and combines $M$ branches, SRake estimates $M$ channels and combines $L$ paths, while PRake estimates $L$ channels and combines $L$ paths. In the following, the proposed receivers are presented in details.

\section{A. Adaptive SRake}

Fig. 1 describes the operation scheme of the ASRake receiver. More specifically, ASRake receiver introduces two parameters, which control the number of the "significant" paths that will be taken into account. The first parameter is the threshold, $\gamma_{T}$, which must be reached by the sum of the SNR of the selected paths. The second parameter, $\mu$, controls the performance improvement that each path should provide in case of selection. Both conditions lead in breaking of the selection process. To sum up, the receiver keeps adding paths only if the sum of their SNR has not reached the threshold $\gamma_{T}$ and the ratio of each branch (see Fig. 1) to the first one is below $\mu$. ASRake receiver, similar to the SRake one, estimates the instantaneous powers of all the $M$ resolvable paths so that it can sort the $L$ ones according to their powers.

The main advantage of the ASRake receiver is the power consumption reduction, compared to ARake, because it avoids to combine those paths that practically do not offer much in the system performance (i.e. the low SNR paths), or it stops combining paths as soon as the desirable quality has been reached. This is important, especially in diversity rich environments, where the resolvable paths are more than a hundred and combining a fixed number of them results in insufficient exploitation of system resources.

\section{B. Adaptive PRake}

The mode of operation for the APRake receiver is also described in Fig. 1 and is similar to that of the ASRake receiver; that is, the receiver keeps adding paths until either the desired output combined SNR is achieved or the addition of the next path does not compensate the expected performance improvement. However, the APRake receiver estimates considerably less paths, since it combines the first arriving paths and not the strongest ones as the SRake does.

Consequently, APRake receiver offers lower complexity and power consumption, compared to SRake receivers. In comparison to PRake receiver, APRake offers the advantage of adaptation to channel conditions, since it stops estimating and combining branches, as soon as the two predetermined conditions are not fulfilled.

\section{Performance AnAlysis}

In this Section, we evaluate the performance of Rake receivers, well-known in the literature, i.e. SRake, PRake, NT-GSC, MS-GSC and compare them with the proposed ASRake and APRake. The comparison is made in terms of the average Bit Error Probability (ABEP) and the number of combined branches. As mentioned above, we consider realistic UWB channels, and we follow the semi-analytical evaluation of the BEP that was presented in [6]. The maximum number of combined paths for the NT-GSC, MS-GSC and the proposed receivers is set to 16 .
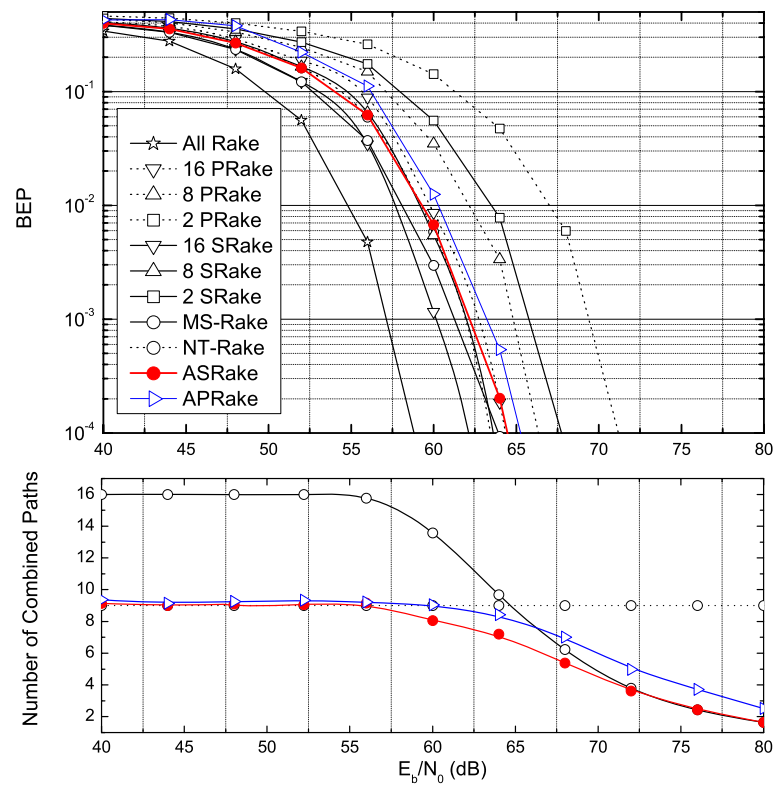

Fig. 2. The ABEP and the number of combined branches versus the first path SNR for the CM1 channel model. 

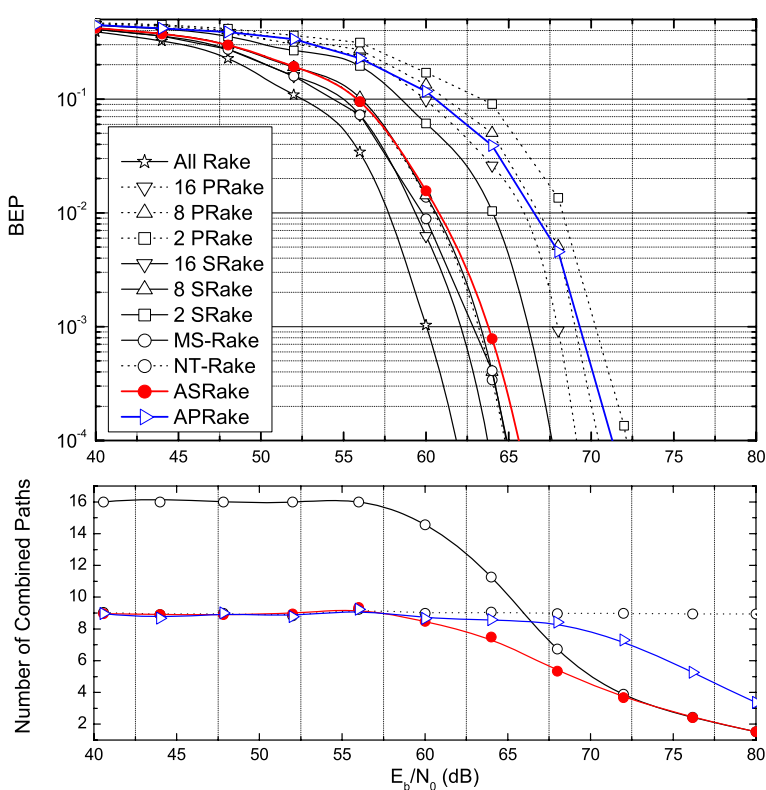

Fig. 3. The ABEP and the number of combined branches versus the first path SNR for the CM2 channel model.
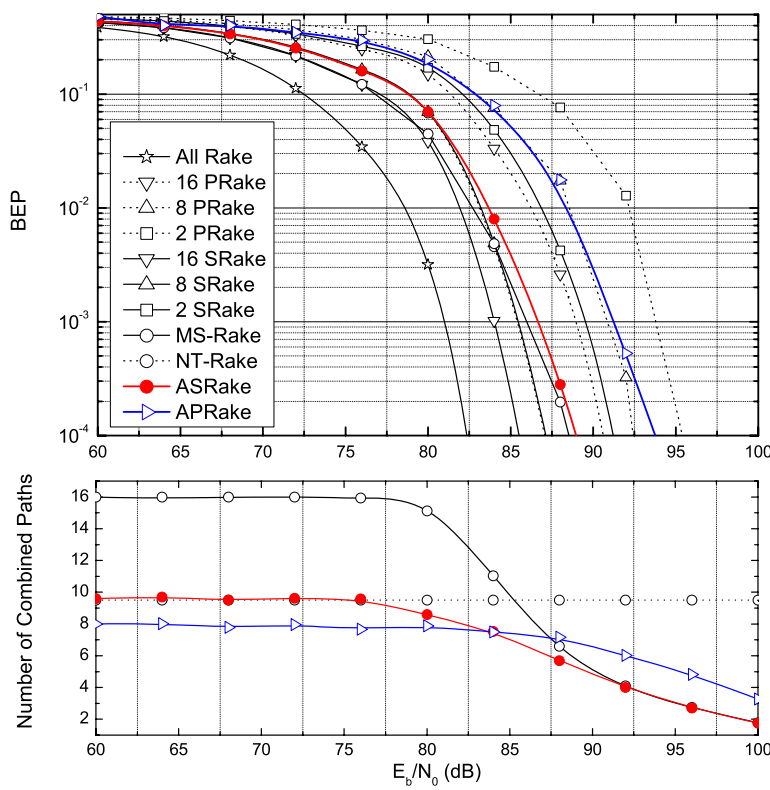

Fig. 4. The ABEP and the number of combined branches versus the first path SNR for the CM3 channel model.

In Fig. 2, the ABEP and the number of the combined branches are plotted against SNR for the CM1 channel model. The threshold, $\gamma_{T}$, has been set to $25 \mathrm{~dB}$ and $\mu=0.6$ and $\mu=0.3$ for the ASRake and APRake, respectively. We note that the performance of the ARake receiver is a lower bound to the ABEP and serves only as a benchmark, since it cannot be implemented in practice. By inspection of this figure, two major conclusions can be drawn:

- the receiver's adaptability to channel conditions results in reduction of the number of the required combined paths without significant performance degradation.

- the simultaneous adaptability to more than one criterion (e.g. the output combined SNR, each path's SNR) further reduces the number of the combined paths.

More specifically, we can observe that the receivers that take into account quality criteria, i.e. the NT-GSC, MS-GSC and the proposed ASRake and APRake receivers, combine less paths under certain conditions than the SRake and PRake receivers. For example, NT-GSC combines in average 9 paths and has an ABEP degradation of less than $2 \mathrm{~dB}$, compared to the 16-SRake. Similarly, the proposed ASRake receiver achieves a performance close to that of 16-SRake by combining in average less than the half paths compared to the latter. However, the receivers that adjust the number of their combined branches using one criterion (i.e. the MSGSC and the NT-GSC), have some disadvantages. For example, when using the NT-GSC, the selected paths (i.e., those that satisfy the test per branch rule) can be unnecessarily too many, while a sufficient quality of communication could be possibly attained by combining less paths (e.g. for SNR greater than $55 \mathrm{~dB}$ ).

On the other hand, MS-GSC keeps adding weak paths in its attempt to reach the predetermined threshold, while a slightly worse or the same performance can be achieved by combining only the strong paths (e.g. for SNR less than $67 \mathrm{~dB}$ ). These disadvantages can be efficiently opposed by taking into account the quality of both the output SNR and the SNR of each individual path as in the case of the proposed receivers. We can see that for the whole SNR range ASRake uses always less or equal number of paths compared to MS and NT-GSC, with performance degradation less than $1 \mathrm{~dB}$. In other words, the proposed receivers achieve better adaptation to channel conditions for a predetermined required quality of communication.

The results are also important for the case of the APRake receiver. The reduction in the number of the combined paths in combination with the fact that PRake estimates the power of the first arriving paths (in contrast to SRake), results in a Rake receiver with lower complexity. Regarding the impact of the ratio of the two indexes, the number of the combined branches, and as a result the offered quality, is increased with the threshold and decreased with the augmentation of $\mu$.

The results in Fig. 3 are based on the CM2 channel model, which is a non line-of sight (NLOS) model. The threshold $\gamma_{T}$ has been set to $25 \mathrm{~dB}$ and $\mu=0.45$ and $\mu=0.01$ for the ASRake and APRake respectively. Regarding the SRake receivers, the conclusions do not differ from those related to the CM1 model. However, we can observe that in this case the PRake receivers achieve considerably worse performance, since in NLOS environments the scattering is severe and the probability that the first arriving paths are the strongest ones is very low. Fig. 4 shows the same results for the CM3 model, where the threshold $\gamma_{T}$ has been set to 25 $\mathrm{dB}$ and $\mu=0.6$ and $\mu=0.2$ for the ASRake and APRake, respectively. The values of the system parameters $\gamma_{T}$ and $\mu$ depend on the quality requirements and the wireless channel. Note, that for the case of the PRake and the CM2 model, $\mu$ has been set to a lower value than that for the CM3 case. This is highly related with the arrival rate of path within each cluster, which is lower for the CM2 model. As a result the difference in power between two sequential paths is small. 

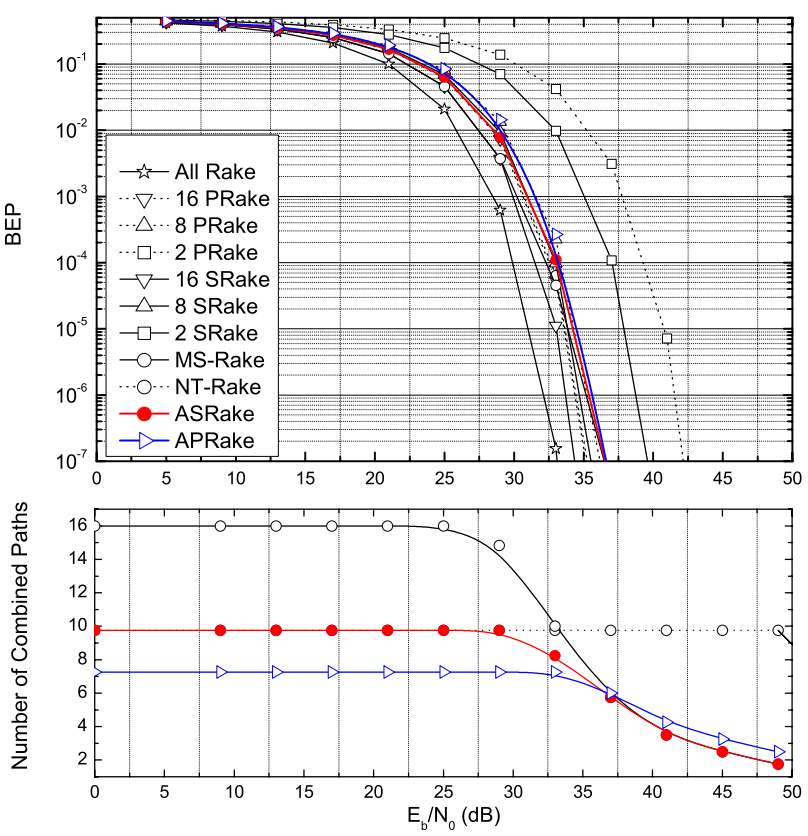

Fig. 5. The ABEP and the number of combined branches versus the first path SNR for the LF channel model.

Finally, Fig. 5 shows the BEP for the case of the LF channel model $\left(\gamma_{T}=25 \mathrm{~dB}\right.$ and $\left.\mu=0.55\right)$. The results are interesting, since as it can been seen, the proposed receivers combine considerably less paths than the other receivers, with performance loss smaller than $2 \mathrm{~dB}$ compared to the 16-SRake. Furthermore, APRake achieves a performance similar to that of 16-SRake, by combining always less than 7 paths. This concludes that in LF channels there is a high probability of combining unnecessarily too many paths, resulting in wasting power and computational resources. Additionally, APRake does not require power estimation of all the resolvable paths, since it only combines the $L$ first arriving ones. Therefore, in LF UWB channels, APRake would be a low complexity and efficient receiver, which achieves the desired quality with minimal resources

\section{CONCLUSIONS}

Two adaptive Rake receivers for UWB applications, called ASRake and APRake, were presented and analyzed. The proposed schemes achieve better adaptation to channel conditions compared to other previously known receivers, without further increasing the complexity. Their performances were evaluated in various practical UWB channels, whose models are based on extensive propagation measurements. The suggested receivers compromise between the hardware complexity, power consumption and performance gain, resulting in significant savings in power and computational resources.

\section{REFERENCES}

[1] M. Z. Win and Z. A. Kostić, "Virtual path analysis of selective Rake receiver in dense multipath channels," IEEE Commun. Lett., vol. 3, pp. 308-310, Nov. 1999.

[2] M. Z. Win, G. Chrisikos, and N. R. Sollenberger, "Performance of Rake reception in dense multipath channels: implications of spreding bandwidth and selection diversity order," IEEE J. Select. Areas Commun., vol. 18, pp. 1516-1525, Aug. 2000.

[3] M. Z. Win and G. Chrisikos, Wideband Wireless Digital Communications, ch. Impact of spreading bandwidth and selection diversity order on selective Rake reception. U.K.: Prentice-Hall, 2001, A. F. Molisch(ed.).

[4] J. D. Choi and W. E. Stark, "Performance of ultra-wideband communications with suboptimal receivers in multipath channels," IEEE J. Select. Areas Commun., vol. 20, pp. 1754-1766, Dec. 2002.

[5] M. Z. Win and R. A. Scholtz, "On the energy capture of ultra -wide bandwidth signals in dense multipath environments," IEEE Commun. Lett., vol. 2, pp. 245-247, Sept. 1998.

[6] Cassioli, D.; Win, M.Z.; Vatalaro, F.; Molisch, A.F. "Low Complexity Rake Receivers in Ultra-Wideband Channels", IEEE Trans. Wireless Commun, vol. 6, pp. 1265-1275, April 2007.

[7] M.K. Simon, and M.-S. Alouini, "Performance analysis of generalized selection combining with threshold test per branch (T-GSC)," IEEE Trans. Vehic. Tech., vol. 51, pp. 1018-1029, Sept. 2002.

[8] P. Gupta, N. Bansal, and R. K. Mallik, "Analysis of minimum selection H-S/MRC in Rayleigh fading," IEEE Trans. Commun., vol. 53, no. 5, pp. 780-784, May 2005.

[9] R. K. Mallik, P. Gupta, and Q. T. Zhang, "Minimum selection GSC in independent Rayleigh fading," IEEE Trans. Veh. Technol., vol. 54, no. 3, pp. 1013-1021, May 2005.

[10] H.-C. Yang, "New results on ordered statitics and analysis of minimum selection generalized selection combining (GSC)," IEEE Trans. Wireless Commun., vol. 5, no. 7, pp. 1876-1885, July 2006.

[11] M.-S. Alouini and H.-C. Yang, "Minimum estimation and combining generalized selection combining (MEC-GSC)," IEEE Trans. Wireless Commun., vol. 6, pp. 526-532, Feb. 2007.

[12] W. Li, J. Zhong and T. A. Gulliver, "A Low Complexity RAKE Receiver for Ultra-Wideband Systems," IEEE Vehicular. Technology Conference., vol. 3, pp. 1393-1396, Sept. 2005.

[13] A. F. Molisch, J. R. Foerster, and M. Pendergrass, "Channel models for ultrawideband personal area networks," IEEE Pers. Commun. Mag., vol. 10, pp. 14-21, Dec. 2003.

[14] D. Cassioli, M. Z. Win, and A. F. Molisch, "The UWB indoor channel: from statistical model to simulations," IEEE J. Select. Areas Commun., vol. 20, pp. 1247-1257, Aug. 2002.

[15] A. F. Molisch et al., "IEEE 802.15.4a channel model - final report," Nov. 2004.

[16] M. Z. Win, R. A. Scholtz, and M. A. Barnes, "Ultra -wide bandwidth signal propagation for indoor wireless communications," in Proc. IEEE Int. Conf. Commun., vol. 1, pp. 56-60, June 1997, Montreal, CANADA.

[17] M. Z. Win and R. A. Scholtz, "Characterization of ultra-wide bandwidth wireless indoor channels: A communication-theoretic view," IEEE J. Select. Areas Commun., vol. 20, pp. 1613-1627, Dec. 2002.

[18] A. A. Saleh and R. A. Valenzuela, "A statistical model for indoor multipath propagation," IEEE J. Select. Areas Commun., vol. 5, pp. 128-137, Feb. 1987.

[19] J. R. Foerster, "Channel modeling sub-committee report final," in Tech. Rep. P802.15 02/490r1, IEEE 802.15 SG3a, Feb. 2003. 